Mathematical Modelling and Analysis

Volume 20 Number 1, January 2015, 9-29

http://dx.doi.org/10.3846/13926292.2015.996615

(C) Vilnius Gediminas Technical University, 2015
Publisher: Taylor\&Francis and VGTU

http://www.tandfonline.com/TMMA

ISSN: $1392-6292$

eISSN: 1648-3510

\title{
Solving Numerically the Static Maxwell Equations in an Axisymmetric Singular Geometry
}

\author{
Franck Assous ${ }^{a}$ and Irina Raichik ${ }^{b}$ \\ ${ }^{a}$ Ariel University \\ 40700 Ariel, Israel \\ ${ }^{b}$ Bar-Ilan University \\ 52900 Ramat-Gan, Israel \\ E-mail(corresp.): franckassous@netscape.net \\ E-mail: irina.raichik@gmail.com
}

Received December 31, 2012; revised November 5, 2014; published online January 15, 2015

\begin{abstract}
We propose a new numerical method to compute the singular solution of the Maxwell equations in axisymmetric domains, as for example in non convex polygonal domains. As geometrical singularities are mainly related to the space dependent part of the model, we focus on the static field computation. We then introduce a new approach, that consists in decomposing the domain into two or more subdomains, and to derive an ad hoc variational formulation in each subdomain. The interface conditions are then imposed with a method deduced from a Nitsche method coupled with a specific "exchange" approach. An advantage of this domain decomposition method is that it does not require neither overlapping nor iteration process. Another advantage is that no particular mesh refinement is needed near the geometrical singularities. Numerical examples will be shown.
\end{abstract}

Keywords: Maxwell equations, singular geometries, Laplace operator, Nitsche method, domain decomposition.

AMS Subject Classification: 65P25; 77C10.

\section{Introduction}

Electromagnetic phenomena play a very important role nowadays. Maxwell's equations (see $[7,8,9,19,24]$ ) represent one of the most elegant and concise ways to state the fundamentals of electricity and magnetism. A lot of engineering problems today require to model and simulate them numerically. Furthermore, a lot of structures one needs to model have a complex three-dimensional geometry and present a surface with reentrant edges and/or corners. They are called geometrical singularities since they can generate very strong fields that have to be taken into account especially in a numerical approach. 
Besides, even if it is sometimes unavoidable, three-dimensional computations may be very expensive. Reducing the three-dimensional problem to twodimensional computations, by assuming that the geometry, the data and the initial conditions are independent of one of coordinates, reduces the cost of computations, and often gives a quite good approximation of the real three dimensional solution.

Various approaches have been suggested for solving Maxwell equations in a singular domain. To our knowledge, first theoretical works have been proposed by M.Birman and M.Solomyak (see [5,6]). The principle of their approach is to decompose the space of electromagnetic fields into a simple sum of a regular field subspace and the subspace made of gradients of solutions to the Laplace problem. However, from a numerical point of view, this decomposition is not very useful since it is not a direct one.

In 1990s, a new vision on the characterizations of the singularity of the electromagnetic fields was given by M. Costabel and M. Dauge (see $[11,12]$ and references therein), called afterwards electromagnetic singularities. They also accomplished a thorough description of the Maxwell operator in 2D and 3D domain and proved density results. Lately (see [13]), they proposed that the electric field would be fully taken into account without any splitting thanks to a technique which consists of introducing suitable weights in the definition of functional spaces: it allows to capture numerically strong electric fields.

We refer to the work of Bonnet-Ben Dhia, Hazard and Lohrengel (see [14]), for solving the time-harmonic, divergence-free Maxwell equations. But, in practice, they use a truncation function that leads to very slow numerical convergence. Let us also mention comparisons of different existing approaches for solving the 2D Maxwell equations (see $[17,20]$ ).

In this paper, we are interested in the axisymmetric singular geometry case $(r, \theta, z)$. Two-dimensional geometry is achieved by assuming that the domain, the data and the initial conditions are independent of $\theta$, namely $\frac{\partial}{\partial \theta}=0$. This can be viewed as an intermediate between a full three-dimensional problem and a two-dimensional one. Since geometric singularities of the domain (like reentrant corners) have basically an influence on the space part of the equations, we will restrict ourselves to the static problem, by assuming $\frac{\partial}{\partial t}=0$, as also developed in [10]. Then, we use that the singularity of the Maxwell equations is connected to the singular solution to the Laplace operator. Hence, we derive a new ad hoc numerical method to solve this Laplace-like problem. This method will be based on a domain decomposition method, so that it may be supported by the huge bibliography of the well-known domain decomposition methods. It will require the introduction of extended Nitsche method (see [4, 25]) coupled with a specific "exchange" approach in order to handle the transmission conditions of Dirichlet and Neumann type. Note that the proposed domain decomposition method does not require neither overlapping nor iteration process, which makes it efficient from a computational point of view. It will be also shown that no particular mesh refinement will be needed near the geometrical singularities. 


\section{From Maxwell to a Laplace-Like Operator}

\subsection{The Maxwell equations}

Let $\Omega$ be a bounded and simply connected Lipschitz domain, $\Gamma$ its boundary, and $\mathbf{n}$ the unit outward normal to $\Gamma$. The time-dependent Maxwell equations are a set of four partial differential equations that relate the electric field $\mathbf{E}$ and the magnetic induction $\mathbf{B}$ to the charge density $\rho$ and the current density $\mathbf{J}$. If we let $c$ and $\varepsilon_{0}$ be the speed of light and the dielectric permittivity of the vacuum, the Maxwell equations in vacuum read

$$
\begin{aligned}
& \frac{\partial \mathbf{E}}{\partial t}-c^{2} \operatorname{curl} \mathbf{B}=-\frac{1}{\varepsilon_{0}} \mathbf{J}, \\
& \frac{\partial \mathbf{B}}{\partial t}+\operatorname{curl} \mathbf{E}=0, \\
& \operatorname{div} \mathbf{E}=\frac{\rho}{\varepsilon_{0}}, \\
& \operatorname{div} \mathbf{B}=0,
\end{aligned}
$$

these quantities depending on the space variable $\mathbf{x}$ and on the time variable $t$. These equations are supplemented with appropriate boundary conditions. In this paper, we assume that the boundary is a perfect conducting medium or perfect conductor, in which the conductivity is assumed to be "infinite": all electromagnetic fields $\mathbf{E}$ and $\mathbf{B}$ are uniformly equal to zero in such a medium. This ideal situation is often used to model metals. Of course, extension to other boundary conditions is possible. Classically, one models the perfect conductor boundary conditions by

$$
\mathbf{E} \times \mathbf{n}=0 \quad \text { and } \quad \mathbf{B} \cdot \mathbf{n}=0 \text { on the boundary } \Gamma \text {. }
$$

Last, initial conditions are provided, for instance at initial time $t=0$

$$
\mathbf{E}(t=0)=\mathbf{B}(t=0)=0 .
$$

\subsection{Reduction to two-dimensional problems}

We assume now that the bounded domain $\Omega$ is an axisymmetric one, limited by the surface of revolution $\Gamma$. Let us denote their intersections with a meridian half-plane by $\omega$ and $\gamma_{b}$ their intersections with a meridian half-plane (see Fig. 1). One has $\partial \omega:=\gamma=\gamma_{a} \cup \gamma_{b}$, where either $\gamma_{a}=\emptyset$ when $\gamma_{b}$ is a closed contour (i.e. $\Omega$ does not contain the axis), or $\gamma_{a}$ is the segment of the axis lying between the extremities of $\gamma_{b}$. We denote $\boldsymbol{\nu}$ is outward normal, and by $\boldsymbol{\tau}$ the unit tangential vector such that $(\boldsymbol{\tau}, \boldsymbol{\nu})$ is direct. It is natural to consider the cylindrical coordinates $(r, \theta, z)$ for such a domain. A meridian half-plane is defined by the equation $\theta=$ constant, and $(r, z)$ are Cartesian coordinates in this half-plane.

Assuming symmetry of revolution, namely $\partial / \partial \theta=0$, means that the fields are entirely determined by their trace in $\omega$, that is the datum of their value in a meridian half-plane. Applying $\partial / \partial \theta=0$ in the classical formulae for 

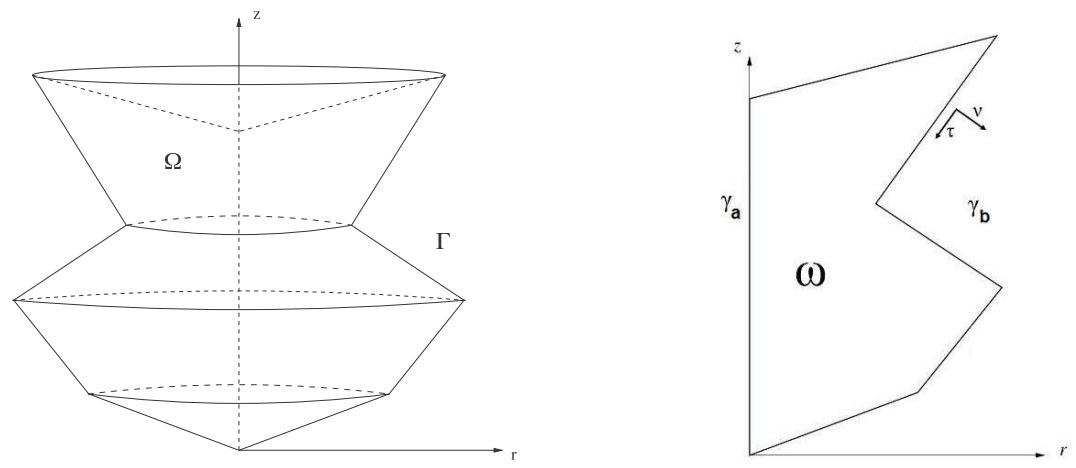

Figure 1. The axisymmetric domain $\Omega$ and the corresponding meridian domain $\omega$.

the gradient, divergence, and curl operators in cylindrical coordinates (see for instance [3]), we get the following expressions:

$$
\begin{gathered}
\operatorname{grad} f=\left(\frac{\partial f}{\partial r}, \frac{\partial f}{\partial z}\right), \quad \operatorname{div} \mathbf{u}=\frac{1}{r} \frac{\partial}{\partial r}\left(r u_{r}\right)+\frac{\partial u_{z}}{\partial z}, \\
\Delta f=\frac{\partial^{2} f}{\partial r^{2}}+\frac{1}{r} \frac{\partial f}{\partial r}+\frac{\partial^{2} f}{\partial z^{2}}
\end{gathered}
$$

whereas the curl operator is given by the formula

$$
\operatorname{curl} \mathbf{u}=\left(-\frac{\partial u_{\theta}}{\partial z}, \frac{\partial u_{r}}{\partial z}-\frac{\partial u_{z}}{\partial r}, \frac{1}{r} \frac{\partial}{\partial r}\left(r u_{\theta}\right)\right) .
$$

Remark that for a meridian vector field $\mathbf{u}_{m}=\left(u_{r}, u_{z}\right)$, its curl curlu $\mathbf{u}_{m}$ is azimuthal, so that it is convenient to introduce the roman type notation

$$
\operatorname{curl} \mathbf{u}_{m}=\frac{\partial u_{r}}{\partial z}-\frac{\partial u_{z}}{\partial r}
$$

In the same way, $\operatorname{curl}_{\theta}$ is meridian if $\mathbf{u}_{\theta}$ is azimuthal. Hence, we set

$$
\operatorname{curl} u_{\theta}=\left(-\frac{\partial u_{\theta}}{\partial z}, \frac{1}{r} \frac{\partial}{\partial r}\left(r u_{\theta}\right) \mathbf{e}_{z}\right) .
$$

As it is well known, these properties lead us to decouple the Maxwell system (2.1)-(2.4) into two systems of equations. From now on, let us denote the meridian component of the electric field and of the current density by $\mathbf{E}=$ $\left(E_{r}, E_{z}\right), \mathbf{J}=\left(J_{r}, J_{z}\right)$. Also denote by $B_{\theta}, J_{\theta}$ the azimuthal component of the magnetic induction and of the current density, the axisymmetric Maxwell equations can be written as a system in $\left(\mathbf{E}, B_{\theta}\right)$

$$
\left\{\begin{array}{l}
\frac{\partial \mathbf{E}}{\partial t}-c^{2} \operatorname{curl} B_{\theta}=-\frac{1}{\varepsilon_{0}} \mathbf{J} \\
\frac{\partial B_{\theta}}{\partial t}+\operatorname{curl} \mathbf{E}=0 \\
\operatorname{div} \mathbf{E}=\frac{\rho}{\varepsilon_{0}}
\end{array}\right.
$$


where the perfect conductor boundary condition is expressed

$$
\mathbf{E} \cdot \boldsymbol{\tau}=0 \quad \text { on } \gamma_{b}
$$

and the symmetry conditions on the axis $\gamma_{a}$ become

$$
\mathbf{E} \cdot \boldsymbol{\nu}=E_{r}=0, \quad B_{\theta}=0 \quad \text { on } \gamma_{a} .
$$

Then, with similar notations, a second system in $\left(\mathbf{B}, E_{\theta}\right)$ reads:

$$
\left\{\begin{array}{l}
\frac{\partial E_{\theta}}{\partial t}-c^{2} \operatorname{curl} \mathbf{B}=-\frac{1}{\varepsilon_{0}} J_{\theta} \\
\frac{\partial \mathbf{B}}{\partial t}+\operatorname{curl} E_{\theta}=0 \\
\operatorname{div} \mathbf{B}=0
\end{array}\right.
$$

with the perfect conductor boundary condition

$$
\mathbf{B} \cdot \boldsymbol{\nu}=0, \quad E_{\theta}=0 \quad \text { on } \gamma_{b},
$$

and the symmetry conditions on the axis $\gamma_{a}$

$$
\mathbf{B} \cdot \boldsymbol{\nu}=B_{r}=0, \quad E_{\theta}=0 \quad \text { on } \gamma_{a} .
$$

The two systems (2.8) and (2.9) are of the same nature. In this paper, we will focus on the second one, in which $\left(E_{\theta}, \mathbf{B}\right)$ are involved. The first one may analysed similarly. Remark however that in that case, the Laplace-like operator involved is not the same, but the one studied in [23].

At this point, let us introduce some Sobolev functional spaces and their respective norms, which will be useful for the following of the article. We define

$$
\begin{aligned}
L_{r}^{2}(\omega) & =\left\{f: \omega \rightarrow \mathbb{R} ; \int_{\omega} f^{2} r d r d z<+\infty\right\}, \quad\|f\|_{0, \omega}=\left(\int_{\omega} f^{2} r d r d z\right)^{1 / 2}, \\
H_{r}^{1}(\omega) & =\left\{f \in L_{r}^{2}(\omega) ; \int_{\omega}|\operatorname{grad} f|^{2} r d r d z<+\infty\right\}, \\
\|f\|_{1, \omega} & =\left(\|f\|_{0, \omega}^{2}+\|\operatorname{grad} f\|_{0, \omega}^{2}\right)^{1 / 2}, \\
H_{m}^{1}(\omega) & =\left\{f \in H_{r}^{1}(\omega) ; \int_{\omega} \frac{f^{2}}{r} d r d z<+\infty\right\}, \\
\|f\|_{m, \omega} & =\left(\|f\|_{1, \omega}^{2}+\left\|\frac{f}{r}\right\|_{0, \omega}^{2}\right)^{1 / 2} .
\end{aligned}
$$

Denoting the vector space $\mathbf{L}_{\mathbf{r}}^{\mathbf{2}}(\omega):=L_{r}^{2}(\omega) \times L_{r}^{2}(\omega)$ allows us to define the classical spaces for the Maxwell equations

$$
\begin{aligned}
\mathbf{H}(\operatorname{curl}, \omega) & =\left\{\mathbf{v} \in \mathbf{L}_{r}^{2}(\omega) ; \operatorname{curl} \mathbf{v} \in L_{r}^{2}(\omega)\right\}, \\
\mathbf{H}(\operatorname{div}, \omega) & =\left\{\mathbf{v} \in \mathbf{L}_{\mathbf{r}}^{\mathbf{2}}(\omega) ; \operatorname{div} \mathbf{v} \in L_{r}^{2}(\omega)\right\} .
\end{aligned}
$$


In the rest of the paper, we will need $\mathbf{H}_{0}(\operatorname{div}, \omega)$, the subspace of $\mathbf{H}(\operatorname{div}, \omega)$ with the vanishing normal trace, and the space of solutions

$$
\mathbf{Y}:=\mathbf{H}(\operatorname{curl}, \omega) \cap \mathbf{H}_{0}(\operatorname{div}, \omega) .
$$

Now, considering the azimuthal component $E_{\theta}$, one can easily show that it is a solution to a scalar wave equation. Indeed, it is sufficient to take the time derivative of the first equation of (2.9), to apply the curl operator to the second one, and to use the identity curl curl $=-\Delta$. In these conditions, even in a singular domain, $E_{\theta}$ is always regular, namely belongs to $H_{m}^{1}(\omega)$ (see for a proof [3]) and there is no difficulty to compute it.

For this reason, we focus on the computation of magnetic field $\mathbf{B}$. Moreover, as we handle the geometrical singularity of the domain, we are dealing with the space-dependent part of the model (not the time-dependent one). Thus, we simplify the problem by considering the stationary problem associated with equations (2.9), by performing $\frac{\partial}{\partial t}=0$, which becomes

$$
\left\{\begin{array}{l}
\operatorname{curl} \mathbf{B}=\frac{1}{c^{2} \varepsilon_{0}} \mathbf{J}_{\theta}, \\
\operatorname{div} \mathbf{B}=0 .
\end{array}\right.
$$

This equation appears as a particular case of the following (div, curl) problem, that we will examine next:

For a given scalar function $f$, find $\mathbf{u}=\left(u_{r}, u_{z}\right)$ a divergence-free vector solution in a meridian singular two dimensional domain $\omega$, solution to

$$
\left\{\begin{array}{l}
\operatorname{curl} \mathbf{u}=f \\
\mathbf{u} \cdot \boldsymbol{\nu}=0
\end{array}\right.
$$

\subsection{A decomposition in regular and singular parts}

Much of this subsection is taken and adapted from $[3, \S 3]$, to make the article self-contained. The reader interested in a practical approach only may skip to Section 3. As proved in [3], problem (2.11) is singular in the sense that for a non-convex domain $\omega$ - typically $\omega$ containing a reentrant corner - the space of solutions, says $\mathbf{W}$, is a subspace of $\mathbf{Y}$ but not a subspace of $\mathbf{H}^{1}(\omega)$. More precisely, $\mathbf{W}$ is defined by

$$
\mathbf{W}=\{\mathbf{w} \in \mathbf{Y}: \operatorname{div} \mathbf{w}=0\}, \quad \text { with norm }\|\operatorname{curl} \mathbf{w}\|_{0, w} .
$$

Nevertheless, $\mathbf{W}$ can be decomposed into two subspaces, where $\oplus$ denotes a direct sum

$$
\mathbf{W}=\mathbf{W}_{R} \oplus \mathbf{W}_{S}
$$

with

$$
\mathbf{W}_{R}=\mathbf{W} \cap \mathbf{H}^{1}(\omega) .
$$


Hence, $\mathbf{W}_{R}$ is a regular subspace, as a subspace of $\mathbf{H}^{1}(\omega)$, and one can compute a numerical approximation by a standard method, for instance a $P_{1}$-conforming finite element method. The difficulty comes from the singular subspace $\mathbf{W}_{S}$, that has been proved to be a finite-dimensional subspace (see [1]), the dimension being the number of reentrant corners of the domain $\omega$. Moreover, one has also proved in [2] that a basis function $\mathbf{w}_{S}$ of $\mathbf{W}_{S}$ can be characterized as the solution to

$$
\begin{aligned}
& \operatorname{curl} \mathbf{w}_{S}=P_{S} \quad \text { in } \omega, \\
& \operatorname{div} \mathbf{w}_{S}=0 \text { in } \omega, \\
& \mathbf{w}_{S} \cdot \boldsymbol{\nu}=0 \text { on } \gamma .
\end{aligned}
$$

Here, the right-hand side $P_{S}$ is singular in the sense that it belongs to $L_{r}^{2}(\omega)$, but not to $H_{r}^{1}(\omega)$, and is solution to the problem

$$
\begin{aligned}
& \Delta^{\prime} P_{S}=0 \quad \text { in } \omega, \\
& P_{S}=0 \text { on } \gamma,
\end{aligned}
$$

where $\Delta^{\prime}$ is a Laplace-like operator defined in axisymmetric coordinates $(r, z)$ by:

$$
\Delta^{\prime} P_{S}:=\frac{\partial^{2} P_{S}}{\partial r^{2}}+\frac{\partial^{2} P_{S}}{\partial z^{2}}+\frac{1}{r} \frac{\partial P_{S}}{\partial r}-\frac{P_{S}}{r^{2}} .
$$

Hence, the key point is to compute $P_{S}$, which can not be solved by a standard finite element method, which would give $P_{S}=0$ as a solution. However, following $[15,16,21,22]$, in the cartesian case, or [1] in the axisymmetric case, there exists a non-vanishing singular solution $P_{S}$ which tends to infinity near the reentrant corner, and which does not belong to $H_{r}^{1}(\omega)$ but belongs to $L_{r}^{2}(\omega)$. Our aim is to propose an efficient numerical method to compute $P_{S}$, from which the singular solution $\mathbf{w}_{S}$ will be easily and (hopefully!) accurately computed.

\section{The Numerical Method}

We will introduce now the numerical method to compute $P_{S}$, based on a domain decomposition approach. For the sake of simplicity, we will consider a non convex domain $\omega$ with only one reentrant corner. As we will see in what follows, this domain decomposition approach does not require neither overlapping nor iteration process. This makes it very efficient from a computational point of view.

\subsection{A domain decomposition method}

The principle of a domain decomposition method is to split the computational domain into several subdomains. Here, we split the domain $\omega$ into two subdomains $\omega_{1}$ and $\omega_{2}$. As shown on Figure 2, the subdomain $\omega_{1}$ is taken as the "external" domain and the subdomain $\omega_{2}$ is the vicinity of the reentrant corner, with the interface $\gamma=\omega_{2} \cap \omega_{1}$. For simplicity, we choose $\omega_{2}$ to be an open angular sector in the neighbourhood of the reentrant corner. This leads us to 


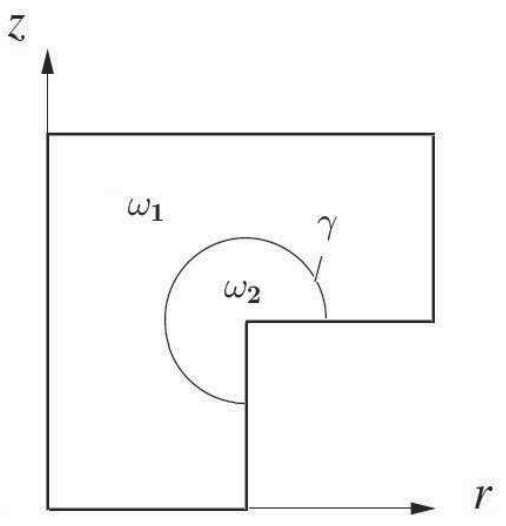

Figure 2. L-shape domain with one reentrant corner.

use local polar coordinates centered at the reentrant corner. In these conditions, $\omega_{1}$ is the "regular" subdomain, since it does not contain the singularity, whereas $\omega_{2}$ is the "singular" subdomain that contains the singularity.

Our aim is now to solve the problem in each subdomain separately, and to take into account the continuity of the solution at the interface $\gamma$. Since we deal with the second order operator $\Delta^{\prime}$, it is necessary to ensure the continuity of the solution $P_{S}$

$$
\left.P_{S}\right|_{\omega_{1}}=\left.P_{S}\right|_{\omega_{2}} \text { across the interface } \gamma
$$

and the continuity of the normal derivative of $P_{S}$

$$
\left.\frac{\partial P_{S}}{\partial \nu_{1}}\right|_{\omega_{1}}+\left.\frac{\partial P_{S}}{\partial \nu_{2}}\right|_{\omega_{2}}=0 \text { across the interface } \gamma
$$

where $\nu_{1}$ and $\nu_{2}$ are respectively the outgoing normals of $\omega_{1}$ and $\omega_{2}$ at the interface $\gamma$. Denoting by $P_{1}$ the "external" solution, that is the restriction of $P_{S}$ to $\omega_{1}$, and by $P_{2}$ the "singular" solution, that is the restriction of $P_{S}$ to $\omega_{2}$, the system (2.15)-(2.16) can be decomposed in the following two systems:

Find $P_{1} \in H_{m}^{1}\left(\omega_{1}\right)$ solution to $\quad$ Find $P_{2} \in H_{m}^{1}\left(\omega_{2}\right)$ solution to

$$
\left\{\begin{array} { l l } 
{ - \Delta ^ { \prime } P _ { 1 } = 0 } & { \text { in } \omega _ { 1 } , } \\
{ P _ { 1 } = 0 } & { \text { on } \partial \omega _ { 1 } , } \\
{ P _ { 1 } = P _ { 2 } } & { \text { on } \gamma , } \\
{ \frac { \partial P _ { 1 } } { \partial \nu _ { 1 } } = - \frac { \partial P _ { 2 } } { \partial \nu _ { 2 } } } & { \text { on } \gamma ; }
\end{array} \quad \left\{\begin{array}{ll}
-\Delta^{\prime} P_{2}=0 & \text { in } \omega_{2}, \\
P_{2}=0 & \text { on } \partial \omega_{2}, \\
P_{2}=P_{1} & \text { on } \gamma, \\
\frac{\partial P_{2}}{\partial \nu_{2}}=-\frac{\partial P_{1}}{\partial \nu_{1}} & \text { on } \gamma .
\end{array}\right.\right.
$$

In the system on $P_{1}$ (left), $P_{2}$ is assumed to be given, whereas in the system on $P_{2}$ (right), $P_{1}$ is assumed to be given. In the regular subdomain $\omega_{1}, P_{1}$ is regular everywhere and can be solved by standard finite element method. However, the 
problem set in $\omega_{2}$ remains singular, since $\omega_{2}$ still contains the reentrant corner, and it would also give $P_{2}=0$ as solution with a standard numerical method. To overcome this difficulty, one uses as in [2] that $P_{2}$ can be decomposed into

$$
P_{2}=Q_{2}+P_{2}^{S}
$$

where $Q_{2}$ is the regular part everywhere in $\omega_{2}$ of $P_{2}$, that is $Q_{2}$ belongs to $H_{r}^{1}\left(\omega_{2}\right) . P_{2}^{S}$ denoting the singular part of $P_{2}$, that is the part that belongs to $L_{r}^{2}\left(\omega_{2}\right)$ but not to $H_{r}^{1}\left(\omega_{2}\right)$. Following [1], we have an analytic expression of the singular part $P_{2}^{S}$, given by

$$
P_{2}^{S}=\frac{r}{a} \rho^{-\alpha} \sin (\alpha \phi),
$$

where $a$ is the distance from the reentrant corner to $z$-axis, and $(\rho, \phi)$ denote the local polar coordinates centered at the reentrant corner. Decomposition (3.1)-(3.2) will be used to derive the final variational formulation. Moreover, we have also to achieve first continuity of the solution. This will be performed by a Nitsche method. Secondly, the continuity of the normal derivative of the solution which will be imposed by an exchange technique in the variational formulations, that will be explained below. Let us introduce now the classical Nitsche method.

\subsection{A variational formulation deduced from a Nitsche method}

In 1970, J. Nitsche proposed in [25] a variational approach to enforce weakly Dirichlet boundary condition. In a certain sense, the method resembles Lagrange multiplier method, but it possesses better convergence property, ensures the existence and uniqueness of the solution.

Nitsche's method can be extend for imposing essential boundary conditions weakly in the finite element method for approximation of elliptic problems. Roughly speaking, essential boundary conditions, like the Dirichlet boundary conditions, are conditions that have to be imposed in the functional space in which the solution belongs to. At the opposite, natural boundary conditions, like the Neumann conditions, are conditions that appear naturally in the variational formulation and that cannot be handled by a Nitsche approach. The strong advantage of Nitsche's method is that it keeps the convergence rate of the finite element method [26], as opposed to the standard penalty method. Essentially, Nitsche's method imposes the boundary conditions via three boundary terms. Two of them contain the weak form of the normal derivatives of the solution and test functions. These two terms cause the method to be symmetric and consistent. A third term depends on the domain triangulation and causes the method to be stable.

To illustrate the method, we consider the standard Laplace problem. For a given bounded domain $\Omega$ of boundary $\Gamma$, let $H^{1 / 2}(\Gamma)$ be equal to the trace on $\Gamma$ of elements $H^{1}(\Omega)$ and introduce the classical problem

For given functions $f \in L^{2}(\Omega)$ and $g \in H^{1 / 2}(\Gamma)$, find the solution $u$ to

$$
\begin{cases}-\Delta u=f & \text { in } \Omega \\ u=g & \text { on } \Gamma .\end{cases}
$$


We first introduce a shape regular finite element partition $\mathcal{T}_{h}=\cup K$ of the domain $\Omega$. For any element $K$ of the mesh $\mathcal{T}_{h}$, let $P_{k}(K)$ be the space of polynomials of degree $k \geq 1$ on $K$. We denote by $E$ an edge of an element $\mathcal{T}_{h}$ and by $C_{h}$ the trace mesh induced by $\mathcal{T}_{h}$ on the boundary $\Gamma$, that is

$$
C_{h}=\left\{E ; E=K \cap \Gamma, K \in \mathcal{T}_{h}\right\} .
$$

Moreover, we assume that the elements of $C_{h}$ verify the regularity condition

$$
h_{E} \leq C \rho_{E},
$$

where $h_{E}$ is the diameter element $E \in C_{h}$ and $\rho_{E}$ is the maximum diameter of circle inscribed in $E$. Finally, let us introduce the finite element space

$$
V_{h}=\left\{v \in H^{1}(\Omega) ; v_{\mid K} \in P_{k}(K)\right\} .
$$

According to these definitions, Nitsche's method for the above problem consists in the following steps:

- Derivation of the classical variational formulation find $u_{h} \in V_{h}$ such that:

$$
\int_{\Omega} \nabla u_{h} \cdot \nabla v d \Omega-\int_{\Gamma} \frac{\partial u_{h}}{\partial n} v d \Gamma=\int_{\Omega} f v d \Omega, \quad \forall v \in V_{h} .
$$

- Symmetrization by adding the expression

$$
-\int_{\Gamma} \frac{\partial v}{\partial n} u_{h} d \Gamma
$$

to left-hand side of equation and, since $u_{h}=g$ on $\Gamma$, adding the expression

$$
-\int_{\Gamma} \frac{\partial v}{\partial n} g d \Gamma
$$

to the right-hand side of equation, that yields

$$
\int_{\Omega} \nabla u_{h} \cdot \nabla v d \Omega-\int_{\Gamma} \frac{\partial u_{h}}{\partial n} v d \Gamma-\int_{\Gamma} \frac{\partial v}{\partial n} u_{h} d \Gamma=\int_{\Omega} f v d \Omega-\int_{\Gamma} \frac{\partial v}{\partial n} g d \Gamma .
$$

- Stabilization to ensure stability and coerciveness of the above variational formulation, by adding the expression ( $\beta$ being a given constant)

$$
\beta \sum_{E \in C_{h}} \frac{1}{h_{E}} \int_{E} u_{h} v d E
$$

to the left-hand side of equation, and in parallel the expression

$$
\beta \sum_{E \in C_{h}} \frac{1}{h_{E}} \int_{E} g v d E
$$

to the right-hand side of equation. 
Hence, the Nitsche formulation for the Laplace operator is finally written as

$$
\begin{gathered}
\int_{\Omega} \nabla u_{h} \cdot \nabla v d \Omega-\int_{\Gamma} \frac{\partial u_{h}}{\partial n} v d \Gamma-\int_{\Gamma} \frac{\partial v}{\partial n} u_{h} d \Gamma+\beta \sum_{E \in C_{h}} \frac{1}{h_{E}} \int_{E} u_{h} v d E \\
=\int_{\Omega} f v d \Omega-\int_{\Gamma} \frac{\partial v}{\partial n} g d \Gamma+\beta \sum_{E \in C_{h}} \frac{1}{h_{E}} \int_{E} g v d E
\end{gathered}
$$

The method is proved to be consistent and stable for a $\beta$ sufficiently large see [26]. In other words, $u$ solution to (3.3) is also solution to (3.4). Another property of Nitsche's method is the optimal order of convergence. Indeed, Nitsche proved [25] that if $\beta$ is a sufficently large constant, then the discrete solution converges to the exact one with optimal order in $H^{1}$ and $L^{2}$. Following [26], we introduce the mesh dependent norm $\|v\|_{1, h}$ defined by (with obvious standard notations)

$$
\|v\|_{1, h}=\left(\|\nabla v\|_{0}^{2}+\sum_{E \in C_{h}} h_{E}^{-1}\|v\|_{0, E}^{2}\right)^{1 / 2} .
$$

Assuming that $u \in H^{k+1}(\omega)$ and that $\beta$ is sufficiently large, then it holds for $u_{h} \in V_{h}$ the solution to the Nitsche problem (3.4)

$$
\left\|u-u_{h}\right\|_{1, h} \leq C h^{k}\|u\|_{k+1},
$$

where $C$ is a positive constant.

\subsection{Computation of the basis $P_{S}$}

The first step consists in applying the Nitsche method here to impose $P_{1}=P_{2}$ on $\gamma$ for subdomain $\omega_{1}, P_{2}$ being considered as a data, and reciprocally. We get the two following variational formulations:

Find $P_{1} \in H_{m}^{1}\left(\omega_{1}\right)$ solution to

$$
\begin{aligned}
\iint_{\omega_{1}} & \left(\nabla P_{1} \nabla \varphi_{1}+\frac{P_{1} \varphi_{1}}{r^{2}}\right) r d \omega \\
& -\int_{\gamma} \frac{\partial P_{1}}{\partial \nu_{1}} \varphi_{1} r d \gamma-\int_{\gamma} \frac{\partial \varphi_{1}}{\partial \nu_{1}} P_{1} r d \gamma+\beta \sum_{E \in \sum_{h}} \frac{1}{h_{E}} \int_{E} P_{1} \varphi_{1} r d E \\
= & -\int_{\gamma} \frac{\partial \varphi_{1}}{\partial \nu_{1}} P_{2} r d \gamma+\beta \sum_{E \in \sum_{h}} \frac{1}{h_{E}} \int_{E} P_{2} \varphi_{1} r d E, \quad \forall \varphi_{1} \in H_{m 0}^{1}\left(\omega_{1}\right) ;
\end{aligned}
$$

Find $P_{2} \in H_{m}^{1}\left(\omega_{2}\right)$ solution to

$$
\begin{aligned}
& \iint_{\omega_{2}}\left(\nabla P_{2} \nabla \varphi_{2}+\frac{P_{2} \varphi_{2}}{r^{2}}\right) r d \omega-\int_{\gamma} \frac{\partial P_{2}}{\partial \nu_{2}} \varphi_{2} r d \gamma \\
& \quad-\int_{\gamma} \frac{\partial \varphi_{2}}{\partial \nu_{2}} P_{2} r d \gamma+\beta \sum_{E \in \sum_{h}} \frac{1}{h_{E}} \int_{E} P_{2} \varphi_{2} r d E \\
& =-\int_{\gamma} \frac{\partial \varphi_{2}}{\partial \nu_{2}} P_{1} r d \gamma+\beta \sum_{E \in \sum_{h}} \frac{1}{h_{E}} \int_{E} P_{1} \varphi_{2} r d E, \quad \forall \varphi_{2} \in H_{m 0}^{1}\left(\omega_{2}\right) .
\end{aligned}
$$


In addition, we have now to impose the continuity of the normal derivatives across $\gamma$, that the Nistche method does not ensure. To deal with this problem, we introduce an exchange method that consists in exchanging the term $\partial P_{1} / \partial \nu_{1}$ with $-\partial P_{2} / \partial \nu_{2}$ in the variational formulation on $\omega_{1}$, and reciprocally for the variational formulation on $\omega_{2}$. The above variational formulations become:

Find $P_{1} \in H_{m}^{1}\left(\omega_{1}\right)$ solution to

$$
\begin{aligned}
\iint_{\omega_{1}} & \left(\nabla P_{1} \nabla \varphi_{1}+\frac{P_{1} \varphi_{1}}{r^{2}}\right) r d \omega+\int_{\gamma} \frac{\partial P_{2}}{\partial \nu_{2}} \varphi_{1} r d \gamma \\
& -\int_{\gamma} \frac{\partial \varphi_{1}}{\partial \nu_{1}} P_{1} r d \gamma+\beta \sum_{E \in \sum_{h}} \frac{1}{h_{E}} \int_{E} P_{1} \varphi_{1} r d E \\
= & -\int_{\gamma} \frac{\partial \varphi_{1}}{\partial \nu_{1}} P_{2} r d \gamma+\beta \sum_{E \in \sum_{h}} \frac{1}{h_{E}} \int_{E} P_{2} \varphi_{1} r d E, \quad \forall \varphi_{1} \in H_{m 0}^{1}\left(\omega_{1}\right) ;
\end{aligned}
$$

Find $P_{2} \in H_{m}^{1}\left(\omega_{2}\right)$ solution to

$$
\begin{aligned}
\iint_{\omega_{2}} & \left(\nabla P_{2} \nabla \varphi_{2}+\frac{P_{2} \varphi_{2}}{r^{2}}\right) r d \omega+\int_{\gamma} \frac{\partial P_{1}}{\partial \nu_{1}} \varphi_{2} r d \gamma \\
& -\int_{\gamma} \frac{\partial \varphi_{2}}{\partial \nu_{2}} P_{2} r d \gamma+\beta \sum_{E \in \sum_{h}} \frac{1}{h_{E}} \int_{E} P_{2} \varphi_{2} r d E \\
= & -\int_{\gamma} \frac{\partial \varphi_{2}}{\partial \nu_{2}} P_{1} r d \gamma+\beta \sum_{E \in \sum_{h}} \frac{1}{h_{E}} \int_{E} P_{1} \varphi_{2} r d E, \quad \forall \varphi_{2} \in H_{m 0}^{1}\left(\omega_{2}\right) .
\end{aligned}
$$

As explained above, we must use the substitution (3.1) at this point. Indeed numerically solving the above formulation with a standard $P_{k}$-conforming finite element method would give $P_{2} \equiv 0$ in the vicinity $\omega_{2}$ of the reentrant corner. Hence, due to the continuity conditions, one would also get $P_{1} \equiv 0$ in the subdomain $\omega_{1}$, that is $P \equiv 0$ in the whole domain $\omega$. Moreover, the order of convergence of the method (see (3.5)) is not valid in $\omega_{2}$, due to the lack of regularity of $P_{2}$. However, it is valid for its regular part $Q_{2}$. We get

$$
\frac{\partial P_{2}}{\partial \nu_{2}}=\frac{\partial Q_{2}}{\partial \nu_{2}}+\frac{\partial}{\partial \nu_{2}}\left(\frac{r}{a} \rho^{-\alpha} \sin (\alpha \phi)\right) .
$$

Moreover, using that

$$
\Delta^{\prime}\left(\frac{r}{a} \rho^{-\alpha} \sin (\alpha \phi)\right)=\frac{-3 \alpha}{a} \rho^{-\alpha-1} \sin ((\alpha+1) \phi),
$$

we are now looking for $Q_{2}$ - instead of $P_{2}$ - satisfying

$$
\left\{\begin{array}{l}
-\Delta Q_{2}=\frac{-3 \alpha}{a} \rho^{-\alpha-1} \sin ((\alpha+1) \phi) \quad \text { in } \omega_{2}, \\
Q_{2}=0 \quad \text { on } \partial \omega_{2}, \\
Q_{2}=P_{1}-\frac{r}{a} \rho^{-\alpha} \sin (\alpha \phi) \quad \text { on } \gamma, \\
\frac{\partial Q_{2}}{\partial \nu_{2}}=-\frac{\partial P_{1}}{\partial \nu_{1}}-\frac{\partial}{\partial \nu_{2}}\left(\frac{r}{a} \rho^{-\alpha} \sin (\alpha \phi)\right) \quad \text { on } \gamma .
\end{array}\right.
$$


In these conditions, we obtain a new variational formulation with $\left(P_{1}, Q_{2}\right)$ as unknowns, that reads

Find $P_{1} \in H_{m}^{1}\left(\omega_{1}\right)$ such that

$$
\begin{aligned}
\iint_{\omega_{1}} & \left(\nabla P_{1} \nabla \varphi_{1}+\frac{P_{1} \varphi_{1}}{r^{2}}\right) r d \omega+\int_{\gamma} \frac{\partial Q_{2}}{\partial \nu_{2}} \varphi_{1} r d \gamma \\
& -\int_{\gamma} \frac{\partial \varphi_{1}}{\partial \nu_{1}} P_{1} r d \gamma+\beta \sum_{E \in \sum_{h}} \frac{1}{h_{E}} \int_{E} P_{1} \varphi_{1} r d E \\
= & -\int_{\gamma} \frac{\partial}{\partial \nu_{2}}\left(\frac{r}{a} \rho^{-\alpha} \sin (\alpha \phi)\right) \varphi_{1} r d \gamma-\int_{\gamma} \frac{\partial \varphi_{1}}{\partial \nu_{1}} Q_{2} r d \gamma \\
& -\int_{\gamma} \frac{\partial \varphi_{1}}{\partial \nu_{1}} \frac{r}{a} \rho^{-\alpha} \sin (\alpha \phi) r d \gamma+\beta \sum_{E \in \sum_{h}} \frac{1}{h_{E}} \int_{E} Q_{2} \varphi_{1} r d E \\
& +\beta \sum_{E \in \sum_{h}} \frac{1}{h_{E}} \int_{E}\left(\frac{r}{a} \rho^{-\alpha} \sin (\alpha \phi)\right) \varphi_{1} r d E, \quad \forall \varphi_{1} \in H_{m 0}^{1}\left(\omega_{1}\right) ;
\end{aligned}
$$

Find $Q_{2} \in H_{m}^{1}\left(\omega_{2}\right)$ such that

$$
\begin{aligned}
& \iint_{\omega_{2}}\left(\nabla Q_{2} \nabla \varphi_{2}+\frac{Q_{2} \varphi_{2}}{r^{2}}\right) r d \omega+\int_{\gamma} \frac{\partial P_{1}}{\partial \nu_{1}} \varphi_{2} r d \gamma \\
& \quad-\int_{\gamma} \frac{\partial \varphi_{2}}{\partial \nu_{2}} Q_{2} r d \gamma+\beta \sum_{E \in \sum_{h}} \frac{1}{h_{E}} \int_{E} Q_{2} \varphi_{2} r d E \\
& =-\int_{\gamma} \frac{\partial}{\partial \nu_{2}}\left(\frac{r}{a} \rho^{-\alpha} \sin (\alpha \phi)\right) \varphi_{2} r d \gamma-\int_{\gamma} \frac{\partial \varphi_{2}}{\partial \nu_{2}} P_{1} r d \gamma-\int_{\gamma} \frac{\partial \varphi_{2}}{\partial \nu_{2}} \frac{r}{a} \rho^{-\alpha} \sin (\alpha \phi) r d \gamma \\
& \quad+\beta \sum_{E \in \sum_{h}} \frac{1}{h_{E}} \int_{E} P_{1} \varphi_{2} r d E-\beta \sum_{h} \frac{1}{h_{E}} \int_{E} \frac{r}{a} \rho^{-\alpha} \sin (\alpha \phi) \varphi_{2} r d E \\
& \quad-\iint_{\omega_{2}} \frac{3 \alpha}{a} \rho^{-\alpha-1} \sin ((\alpha+1) \phi) \varphi_{2} r d \omega, \quad \forall \varphi_{2} \in H_{m 0}^{1}\left(\omega_{2}\right) .
\end{aligned}
$$

To get the variational formulations which are of practical interest for numerical computations, we first remark that due to the geometry of domain $\omega_{2}$, which is a angular sector, we have $\frac{\partial}{\partial \nu_{2}}=\frac{\partial}{\partial \rho}$. Since $r=a+\rho \cos \phi$, we easily get that $\frac{\partial}{\partial \rho}\left(\frac{r}{a}\right)=\frac{1}{a} \frac{\partial}{\partial \rho}(a+\rho \cos \phi)=\frac{1}{a} \cos \phi$ which leads to

$$
\frac{\partial}{\partial \rho}\left(\frac{r}{a} \rho^{-\alpha} \sin (\alpha \phi)\right)=\left(\frac{1}{a} \cos \phi \rho^{-\alpha}-\alpha \frac{r}{a} \rho^{-\alpha-1}\right) \sin (\alpha \phi) .
$$

Substituting these relations in the expressions above, the variational formulations that will be the basis of the numerical scheme read

Find $P_{1} \in H_{m}^{1}\left(\omega_{1}\right)$ such that

$$
\iint_{\omega_{1}}\left(\nabla P_{1} \nabla \varphi_{1}+\frac{P_{1} \varphi_{1}}{r^{2}}\right) r d \omega+\int_{\gamma} \frac{\partial Q_{2}}{\partial \nu_{2}} \varphi_{1} r d \gamma
$$




$$
\begin{aligned}
& -\int_{\gamma} \frac{\partial \varphi_{1}}{\partial \nu_{1}} P_{1} r d \gamma+\beta \sum_{E \in \sum_{h}} \frac{1}{h_{E}} \int_{\gamma} P_{1} \varphi_{1} r d E \\
= & -\int_{\gamma}\left(\frac{1}{a} \cos \phi \rho^{-\alpha}-\alpha \frac{r}{a} \rho^{-\alpha-1}\right) \sin (\alpha \phi) \varphi_{1} r d \gamma-\int_{\gamma} \frac{\partial \varphi_{1}}{\partial \nu_{1}} Q_{2} r d \gamma \\
& -\int_{\gamma} \frac{\partial \varphi_{1}}{\partial \nu_{1}} \frac{r}{a} \rho^{-\alpha} \sin (\alpha \phi) r d \gamma+\beta \sum_{E \in \sum_{h}} \frac{1}{h_{E}} \int_{\gamma} Q_{2} \varphi_{1} r d E \\
& +\beta \sum_{E \in \sum_{h}} \frac{1}{h_{E}} \int_{\gamma} \frac{r}{a} \rho^{-\alpha} \sin (\alpha \phi) \varphi_{1} r d E, \quad \forall \varphi_{1} \in H_{m 0}^{1}\left(\omega_{1}\right) ;
\end{aligned}
$$

Find $Q_{2} \in H_{m}^{1}\left(\omega_{2}\right)$ such that

$$
\begin{aligned}
\iint_{\omega_{2}} & \left(\nabla Q_{2} \nabla \varphi_{2}+\frac{Q_{2} \varphi_{2}}{r^{2}}\right) r d \omega+\int_{\gamma} \frac{\partial P_{1}}{\partial \nu_{1}} \varphi_{2} r d \gamma \\
& -\int_{\gamma} \frac{\partial \varphi_{2}}{\partial \nu_{2}} Q_{2} r d \gamma+\beta \sum_{E \in \sum_{h}} \frac{1}{h_{E}} \int_{\gamma} Q_{2} \varphi_{2} r d E \\
= & -\int_{\gamma}\left(\frac{1}{a} \cos \phi \rho^{-\alpha}-\alpha \frac{r}{a} \rho^{-\alpha-1}\right) \sin (\alpha \phi) \varphi_{2} r d \gamma-\int_{\gamma} \frac{\partial \varphi_{2}}{\partial \nu_{2}} P_{1} r d \gamma \\
& -\int_{\gamma} \frac{\partial \varphi_{2}}{\partial \nu_{2}} \frac{r}{a} \rho^{-\alpha} \sin (\alpha \phi) r d \gamma+\beta \sum_{E \in \sum_{h}} \frac{1}{h_{E}} \int_{\gamma} P_{1} \varphi_{2} r d E \\
& -\beta \sum_{E \in \sum_{h}} \frac{1}{h_{E}} \int_{\gamma} \frac{r}{a} \rho^{-\alpha} \sin (\alpha \phi) \varphi_{2} r d E \\
& -\iint_{\omega_{2}} \frac{3 \alpha}{a} \rho^{-\alpha-1} \sin ((\alpha+1) \phi) \varphi_{2} r d \omega, \quad \forall \varphi_{2} \in H_{m 0}^{1}\left(\omega_{2}\right) .
\end{aligned}
$$

As a straight consequence of the previous derivations, we readily get the following consistency result

Theorem 1. Let $P_{S}$ be the singular solution of the equations (2.15)-(2.16). Then $P_{S}$ solves the above systems.

Moreover, using the same tools as for the standard Nitsche method, one can show that this modified axisymmetric Nitsche-like method is stable, for a sufficient large $\beta$. Numerical illustrations of this approach will be given in Section 4.

\subsection{Computation of the basis $\mathrm{w}_{S}$}

Assuming that $P_{S}$ has been obtained, our aim is now to compute a basis function $\mathbf{w}_{S}$ of $\mathbf{W}_{S}$ characterized by (2.12)-(2.14). If we want to use the fact that $\mathbf{w}_{S}$ belongs to $\mathbf{W}_{S} \subset \mathbf{H}(\mathrm{curl}, \omega)$ to solve this problem, we can for example transform it into a problem in curlcurl. A second and more convenient way to find $\mathbf{w}_{S}$ is to use its so-called "stream function" $\psi$. Using the following property (see [1]):

$$
\forall \mathbf{w}_{S} \in \mathbf{W}_{S} ; \exists ! \psi \in H_{m}^{1}(\omega) \text { such that } \mathbf{w}_{s}=\operatorname{curl} \psi,
$$


where $\psi$ is the unique solution to the Laplace-like problem

$$
\begin{cases}-\Delta^{\prime} \psi=P_{S} & \text { in } \omega \\ \psi=0 & \text { on } \gamma\end{cases}
$$

As it is more easy to invert a Laplace-like operator than a curl or a curlcurl one, instead of solving (2.12)-(2.14), we chose to solve first the system (3.7) to get $\psi$, then to compute $\mathbf{w}_{S} \in \mathbf{W}_{S}$ solution to $\mathbf{w}_{S}=\operatorname{curl} \psi$. Note also that $\psi$ being equal to $\Delta^{\prime-1}$ of $P_{S} \in L_{r}^{2}(\omega)$, it is quite regular and thus easy to compute.

\subsection{Computation of $\psi$}

Using the same domain decomposition as for $P_{S}$, we introduce for $\psi_{S}$ the two systems

Find $\psi_{1} \in H_{m}^{1}\left(\omega_{1}\right)$ solution to $\quad$ Find $\psi_{2} \in H_{m}^{1}\left(\omega_{2}\right)$ solution to

$$
\left\{\begin{array} { l l } 
{ - \Delta ^ { \prime } \psi _ { 1 } = P _ { 1 } } & { \text { in } \omega _ { 1 } , } \\
{ \psi _ { 1 } = 0 } & { \text { on } \partial \omega _ { 1 } , } \\
{ \psi _ { 1 } = \psi _ { 2 } } & { \text { on } \gamma , } \\
{ \frac { \partial \psi _ { 1 } } { \partial \nu _ { 1 } } = - \frac { \partial \psi _ { 2 } } { \partial \nu _ { 2 } } } & { \text { on } \gamma ; }
\end{array} \quad \left\{\begin{array}{ll}
-\Delta^{\prime} \psi_{2}=P_{2} & \text { in } \omega_{2}, \\
\psi_{2}=0 & \text { on } \partial \omega_{2}, \\
\psi_{2}=\psi_{1} & \text { on } \gamma \\
\frac{\partial \psi_{2}}{\partial \nu_{2}}=-\frac{\partial \psi_{1}}{\partial \nu_{1}} & \text { on } \gamma
\end{array}\right.\right.
$$

As previously, one can solve this problem with the variational formulations Find $\psi_{1} \in H_{m}^{1}\left(\omega_{1}\right)$ such that

$$
\begin{array}{r}
\iint_{\omega_{1}}\left(\nabla \psi_{1} \nabla \varphi_{1}+\frac{\psi_{1} \varphi_{1}}{r^{2}}\right) r d \omega-\int_{\gamma} \frac{\partial \psi_{1}}{\partial \nu_{1}} \varphi_{1} r d \gamma \\
-\int_{\gamma} \frac{\partial \varphi_{1}}{\partial \nu_{1}} \psi_{1} r d \gamma+\beta \sum_{E \in \sum_{h}} \frac{1}{h_{E}} \int_{\gamma} \psi_{1} \varphi_{1} r d E \\
=-\int_{\gamma} \frac{\partial \varphi_{1}}{\partial \nu_{1}} \psi_{2} r d \gamma+\beta \sum_{E \in \sum_{h}} \frac{1}{h_{E}} \int_{\gamma} \psi_{2} \varphi_{1} r d E \\
+\iint_{\omega_{1}}\left(P_{1} \varphi_{1}\right) r d \omega, \quad \forall \varphi_{1} \in H_{m 0}^{1}\left(\omega_{1}\right) ;
\end{array}
$$

Find $\psi_{2} \in H_{m}^{1}\left(\omega_{2}\right)$ such that

$$
\begin{aligned}
\iint_{\omega_{2}} & \left(\nabla \psi_{2} \nabla \varphi_{2}+\frac{\psi_{2} \varphi_{2}}{r^{2}}\right) r d \omega-\int_{\gamma} \frac{\partial \psi_{2}}{\partial \nu_{2}} \varphi_{2} r d \gamma \\
- & \int_{\gamma} \frac{\partial \varphi_{2}}{\partial \nu_{2}} \psi_{2} r d \gamma+\beta \sum_{E \in \sum_{h}} \frac{1}{h_{E}} \int_{\gamma} \psi_{2} \varphi_{2} r d E \\
= & -\int_{\gamma} \frac{\partial \varphi_{2}}{\partial \nu_{2}} \psi_{1} r d \gamma+\beta \sum_{E \in \sum_{h}} \frac{1}{h_{E}} \int_{\gamma} \psi_{1} \varphi_{2} r d E \\
& +\iint_{\omega_{2}} P_{2} \varphi_{2} r d \omega, \quad \forall \varphi_{2} \in H_{m 0}^{1}\left(\omega_{2}\right) .
\end{aligned}
$$


As we have already explained in subsection 3.3, after taking into account that $\frac{\partial \psi_{1}}{\partial \nu_{1}}+\frac{\partial \psi_{2}}{\partial \nu_{2}}=0$ and using the exchange method, we get the following final variational formulation

Find $\psi_{1} \in H_{m}^{1}\left(\Omega_{1}\right)$ such that:

$$
\begin{aligned}
\iint_{\omega_{1}} & \left(\nabla \psi_{1} \nabla \varphi_{1}+\frac{\psi_{1} \varphi_{1}}{r^{2}}\right) r d \omega+\int_{\gamma} \frac{\partial \psi_{2}}{\partial \nu_{2}} \varphi_{1} r d \gamma \\
- & \int_{\gamma} \frac{\partial \varphi_{1}}{\partial \nu_{1}} \psi_{1} r d \gamma+\beta \sum_{E \in \sum_{h}} \frac{1}{h_{E}} \int_{\gamma} \psi_{1} \varphi_{1} r d E \\
= & -\int_{\gamma} \frac{\partial \varphi_{1}}{\partial \nu_{1}} \psi_{2} r d \gamma+\beta \sum_{E \in \sum_{h}} \frac{1}{h_{E}} \int_{\gamma} \psi_{2} \varphi_{1} r d E \\
& +\iint_{\omega_{1}}\left(P_{1} \varphi_{1}\right) r d \omega, \quad \forall \varphi_{1} \in H_{m 0}^{1}\left(\omega_{1}\right) ;
\end{aligned}
$$

Find $\psi_{2} \in H_{m}^{1}\left(\Omega_{2}\right)$ such that:

$$
\begin{aligned}
\iint_{\omega_{2}} & \left(\nabla \psi_{2} \nabla \varphi_{2}+\frac{\psi_{2} \varphi_{2}}{r^{2}}\right) r d \omega+\int_{\gamma} \frac{\partial \psi_{1}}{\partial \nu_{1}} \varphi_{2} r d \gamma \\
- & \int_{\gamma} \frac{\partial \varphi_{2}}{\partial \nu_{2}} \psi_{2} r d \gamma+\beta \sum_{E \in \sum_{h}} \frac{1}{h_{E}} \int_{\gamma} \psi_{2} \varphi_{2} r d E \\
= & -\int_{\gamma} \frac{\partial \varphi_{2}}{\partial \nu_{2}} \psi_{1} r d \gamma+\beta \sum_{E \in \sum_{h}} \frac{1}{h_{E}} \int_{\gamma} \psi_{1} \varphi_{2} r d E \\
& +\iint_{\omega_{2}}\left(P_{2} \varphi_{2}\right) r d \omega, \quad \forall \varphi_{2} \in H_{m 0}^{1}\left(\omega_{2}\right) .
\end{aligned}
$$

Remark first that since $\psi$ is regular even in the subdomain $\omega_{2}$, a decomposition similar to (3.1) used for the computation of $P_{S}$ in $\omega_{2}$, even it does exist, is useless. After $\psi$, the last step consists in computing $\mathbf{w}_{S}$ which satisfies the equation

$$
\mathbf{w}_{S}=\operatorname{curl} \psi
$$

Note that with this approach, we ensure that the divergence of $\mathbf{w}_{S}=0$, due to the relation div curl $\equiv 0$. Here again, we use the same domain decomposition of $\omega=\omega_{1} \cup \omega_{2}$. A $P_{1}$-conforming finite element method has been developed, based on the FreeFem++ package [18], to solve this problem. Numerical illustrations are given in the following section.

\section{Numerical Results}

In this section, we present some numerical results to illustrate the efficiency of the proposed method. We consider the 3-D top hat domain $\Omega$ with a reentrant circular edge, that corresponds to an L-shaped 2-D domain $\omega$ with reentrant corner (see Fig. 2). Our aim is to compute the magnetic basis $\mathbf{w}_{S}$ of $\mathbf{W}_{S}$. 

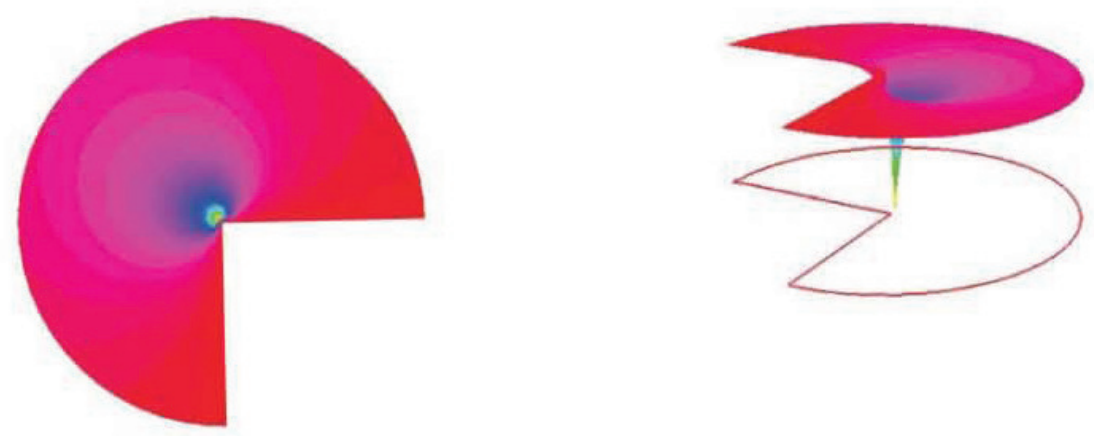

Figure 3. Singular $P_{S}$ solution in the internal domain $\omega_{2}: 2$-d view (left) and 3-d view (right).
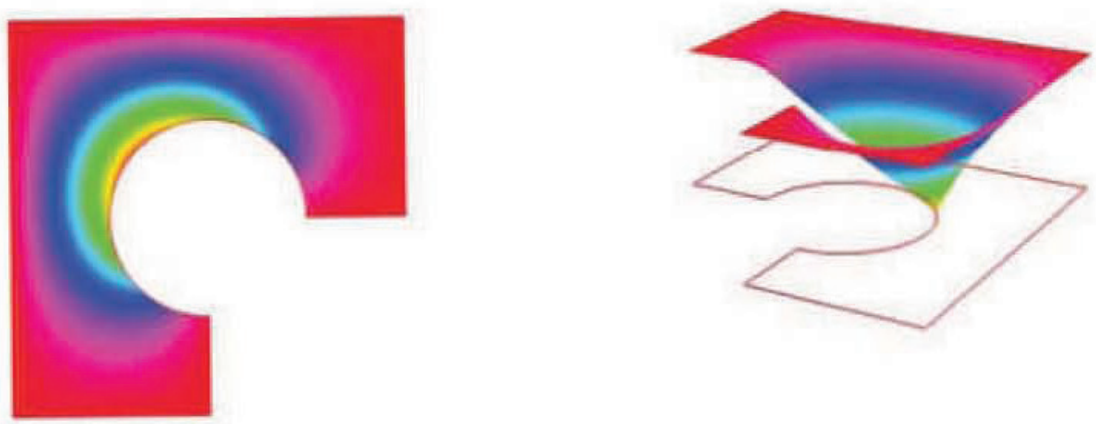

Figure 4. Singular $P_{S}$ solution in the external domain $\omega_{1}: 2$-d view (left) and 3-d view (right).
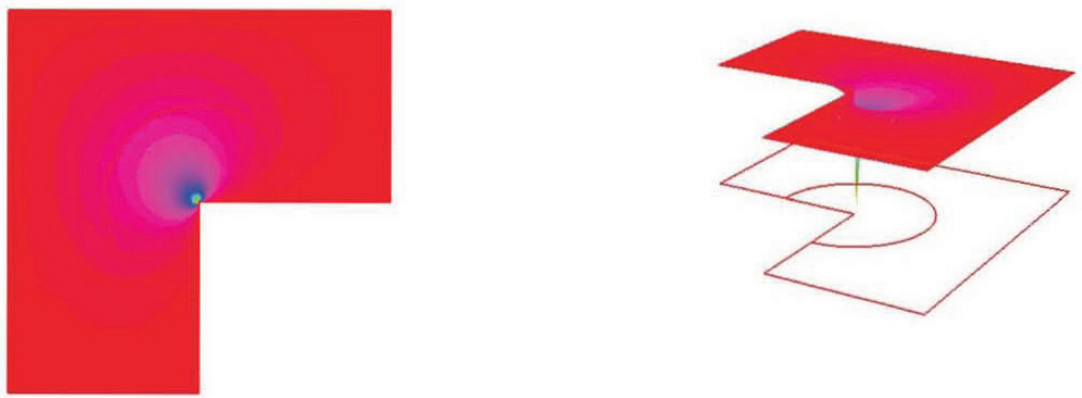

Figure 5. Singular $P_{S}$ solution in the whole domain $\omega$ : 2-d view (left) and 3-d view (right). 

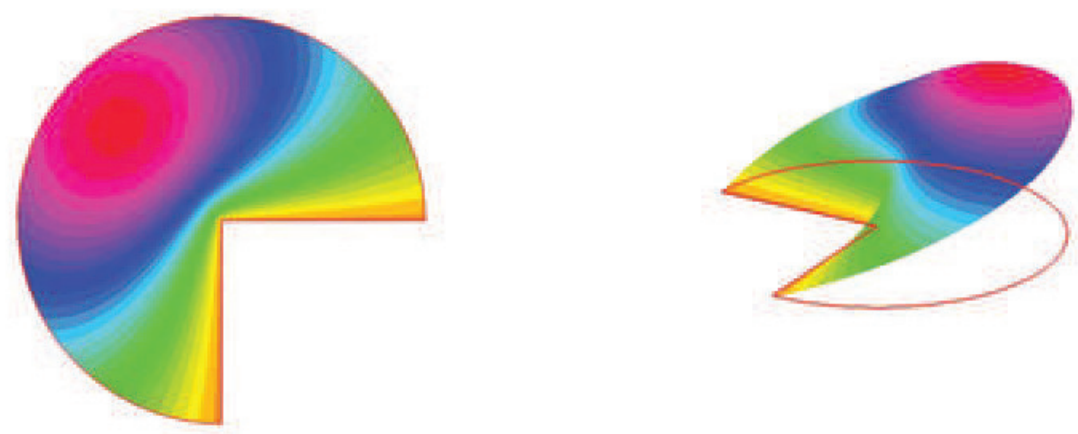

Figure 6. Stream function $\psi$ in the internal domain $\omega_{2}: 2$-d view (left) and 3 -d view (right).
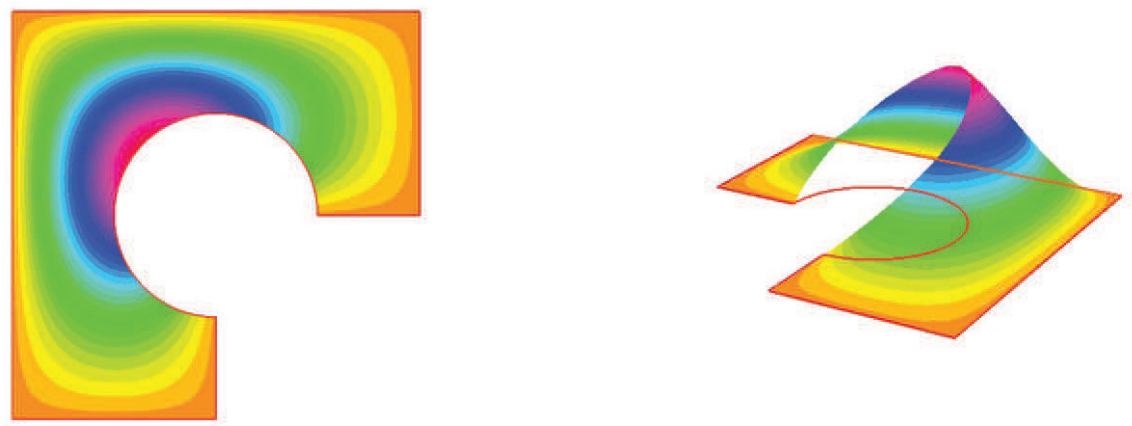

Figure 7. Stream function $\psi$ in the external domain $\omega_{1}: 2$-d view (left) and 3 -d view (right).
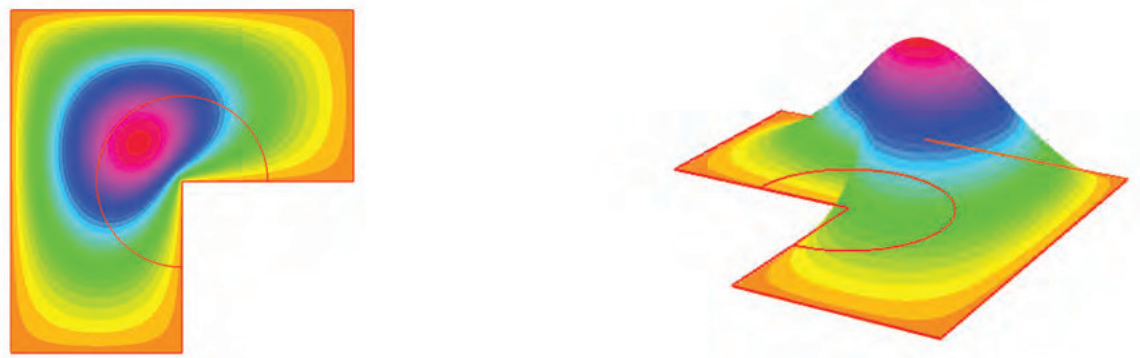

Figure 8. Stream function $\psi$ in the whole domain $\omega$ : 2-d view (left) and 3-d view (right).

We first introduce an unstructured mesh of the L-shaped domain $\omega$ made up of triangles, with no particular mesh refinement near the corner. Following the method exposed in previous sections, to begin with, we compute the singular function $P_{S}$. The numerical results are depicted in Figures 3-5. When representing functions or fields with a singular behavior, we have chosen to truncate 

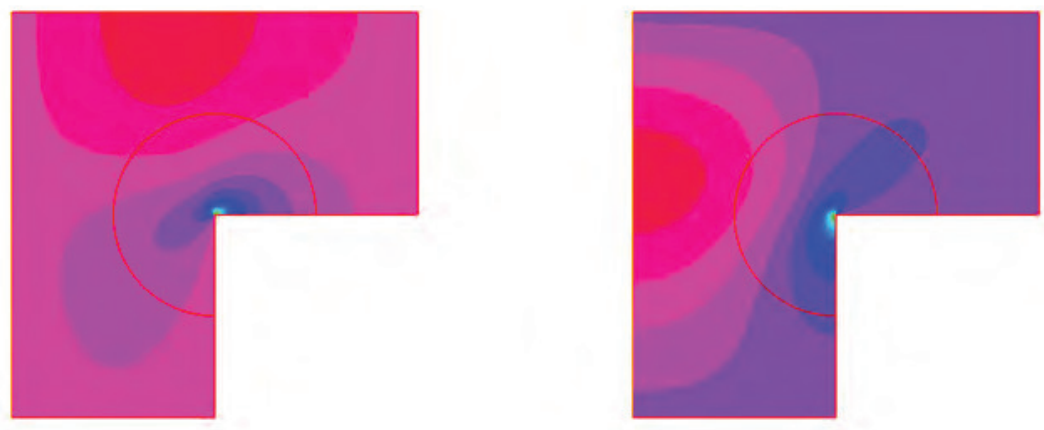

Figure 9. Singular $\mathbf{w}_{S}$ solution: $r$-component (left) and $z$-component (right).

the results in the singular (infinite) node. One can see that the method is able to compute $P_{S}$, whereas a usual method would give $P_{S}=0$ as solution. Moreover, as expected, the continuity of the solution and of its normal derivative are well handled.

The second step consists in computing the stream function $\psi$, where $P_{S}$ is used as a right-hand side. Results are shown in Figures $6-8$, and illustrate that the method is also efficient for more regular functions or fields like $\psi$. Again, the continuity conditions are properly taken into account.

Finally, we get the magnetic basis $\mathbf{w}_{S}$ of $\mathbf{W}_{S}$ by taking the curl of $\psi$, that ensures that the relation $\operatorname{div} \mathbf{w}_{S}=0$. The two components $\left(w_{S}^{r}, w_{S}^{z}\right)$ obtained are depicted in Figure 9. This still shows that the method captures well the field $\mathbf{w}_{S}$ near the edge (and far away from it). A conforming $P^{1}$ Finite Element Method cannot yield such a result. In addition, the results are not noisy, even though the mesh is not particularly refined near the edge.

\section{Conclusion}

In this paper, we presented a new numerical method, based on a domain decomposition approach, to solve the Maxwell equations in an axisymmetric singular domain. It is based on a decomposition of the computational domain into two or more - depending on the number of geometrical singularities - subdomains: an internal one, close to the singularity, and an external one.

Since geometric singularities of the domain have basically an influence on the space part of the equations, we restricted ourselves to the static problem, by assuming $\frac{\partial}{\partial t}=0$. Then, we considered the case of the static magnetic field $\mathbf{B}=\left(B_{r}, B_{z}\right)$.

We have shown that to compute a basis of this singular subspace, the key point is to compute $P_{S}$, which cannot be solved by a standard finite element method, which would give $P_{S}=0$. We proposed a new method to efficiently compute $P_{S}$ and consequently $\mathbf{w}_{S}$. It consists in decomposing the domain $\omega$ into 2 subdomains, and to derive an ad hoc variational formulation, in which the interface conditions have to be handled. 
This method also uses the local expression of the singularity, and an extended version of the Nitsche method, to handle the Dirichlet-type transmission conditions, coupled with a specific "exchange" method, to take into account Neumann-type transmission conditions. An advantage of this domain decomposition method is that it does not require neither overlapping nor iteration process. This makes it efficient from a numerical point of view. Numerical results have been shown to illustrate the method. Moreover, as it is based on on domain decomposition approach, this method can be easily extend to parallel computations.

\section{References}

[1] F. Assous, P. Ciarlet Jr. and S. Labrunie. Theoretical tools to solve the axisymmetric maxwell equations. Math. Meth. Appl. Sci., 25:49-78, 2002. http://dx.doi.org/10.1002/mma.279.

[2] F. Assous, P. Ciarlet Jr. and S. Labrunie. Solution of axisymmetric maxwell equations. Math. Meth. Appl. Sci., 26:861-896, 2003. http://dx.doi.org/10.1002/mma.400.

[3] F. Assous, P. Ciarlet Jr. and J. Segré. Numerical solution to the time-dependent Maxwell equations in two-dimensional singular domain: The singular complement method. J. Comput. Phys., 161:218-249, 2000. http://dx.doi.org/10.1006/jcph.2000.6499.

[4] R. Becker, P. Hansbo and R. Stenberg. A finite element method for domain decomposition with non-matching grids. M2AN, 37:209-225, 2003.

[5] M.Sh. Birman and M.Z. Solomyak. L2-theory of the Maxwell operator in arbitrary domains. Russian Math. Surveys, 42:75-96, 1987. http://dx.doi.org/10.1070/RM1987v042n06ABEH001505.

[6] M.Sh. Birman and M.Z. Solomyak. The weyl asymptotic decomposition of the spectrum of the Maxwell operator for domain with lipschitzian boundary. Vestnik. Leningr. Univ. Math., 20:15-21, 1987.

[7] J. Van Bladel. Electromagnetic Fields. McGraw-Hill, New York, 1985.

[8] M. Cessenat. Mathematical Methods in Electromagnetism. Linear Theory and Applications, volume 41 of Advances in Mathematics for Applied Sciences. World Scientific, Singapore, 1996.

[9] G. Cohen. Higher Order Numerical Methods for Transient Wave Equations. Springer, New York, 2002.

[10] D.M Copeland, J. Gopalakrishnan and J.E. Pasciak. A mixed method for axisymmetric div-curl systems. Math. Comput., 77(264):1941-1965, 2008. http://dx.doi.org/10.1090/S0025-5718-08-02102-9.

[11] M. Costabel. A remark on the regularity of solutions of Maxwell's equations on Lipschitz domains. Math. Meth. Appl. Sci., 12:365-368, 1990. http://dx.doi.org/10.1002/mma.1670120406.

[12] M. Costabel and M. Dauge. Maxwell and Lamé eigenvalues on polyhedral domains. Math. Meth. Appl. Sci., 22:243-258, 1999.

[13] M. Costabel and M. Dauge. Weighted regularization of maxwell equations in polyhedral domains. Numer. Math., 93:239-277, 2002.

http://dx.doi.org/10.1007/s002110100388. 
[14] A.-S. Bonnet-Ben Dhia, C. Hazard and S. Lohrengel. A singular field method for the solution of Maxwell's equations in polyhedral domains. SIAM J. Appl. Math., 59:2028-2044, 1999. http://dx.doi.org/10.1137/S0036139997323383.

[15] P. Grisvard. Elliptic Problems in Nonsmooth Domains, volume 24 of Monogr. Stud. Math. Pitman, London, 1985.

[16] P. Grisvard. Singularities in Boundary Value Problems, volume 22 of RMA. Masson, Paris, 1992.

[17] C. Hazard. Numerical simulation of corner singularities: a paradox in maxwelllike problems. C. R. Acad. Sci. Paris, Ser. IIb, 330:57-68, 2002.

[18] Frédéric Hecht. FreeFem++. Laboratoire J.L. Lions, Université Pierre et Marie Curie, 2010.

[19] J.D. Jackson. Classical Electrodynamics. John Wiley and Sons, New York, 1975.

[20] E. Jamelot. A nodal finite element method for Maxwell's equations. C. R. Acad. Sci. Paris, Ser. I, 339:809-814, 2004.

[21] V.A. Kondrat'ev. The smoothness of solutions of Dirichlet's problem for second order elliptic equation in a region with a piecewise-smooth boundary. Differential Equations, 6:1392-1401, 1976.

[22] V.A. Kondrat'ev. Singularities of a solution of Dirichlet's problem for a secondorder equation in the neighborhood of an edge. Differential Equations, 13:14111415, 1977.

[23] H. Li. Finite element analysis for the axisymmetric laplace operator on polygonal domains. J. Comput. Appl. Math., 235:5155-5176, 2011. http://dx.doi.org/10.1016/j.cam.2011.05.003.

[24] P. Monk. Finite element methods for Maxwell's equations. Oxford University Press, 2003.

[25] J. Nitsche. Über ein Variationsprinzip zur lösung von Dirichlet-Problemen bei Verwendung von Teilrumen, die keinen Randbedingungen unterworfen sind. Abh. Math, Sem. Univ. Hamburg, 36:9-15, 1971.

http://dx.doi.org/10.1007/BF02995904.

[26] R. Stenberg. On some techniques for approximating boundary conditions in the finite element method. J. Comput. Appl. Math., 63:139-148, 1995.

http://dx.doi.org/10.1016/0377-0427(95)00057-7. 